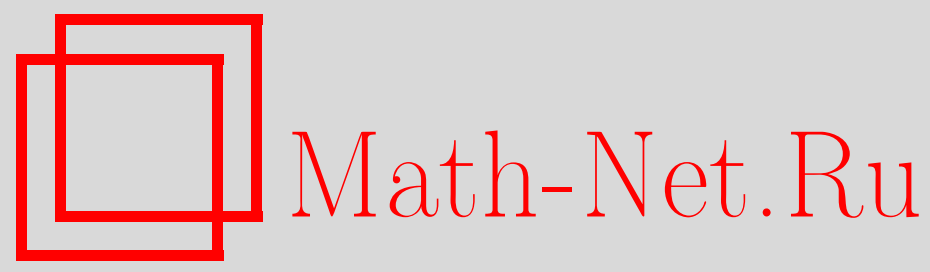

В. Г. Кановей, Нестандартная теория множеств в $\in$-языке, Матем. заметки, 2001, том 70, выпуск 1, 46-50

DOI: https://doi.org/10.4213/mzm717

Использование Общероссийского математического портала Math-Net.Ru подразумевает, что вы прочитали и согласны с пользовательским соглашением http://www . mathnet.ru/rus/agreement

Параметры загрузки:

IP : 54.224 .60 .19

26 апреля 2023 г., 16:25:31

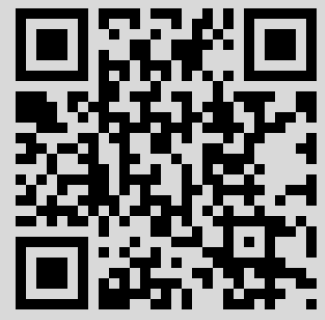


УДК 510.223

\title{
НЕСТАНДАРТНАЯ ТЕОРИЯ МНОЖЕСТВ В $\in$-ЯЗЫКЕ
}

\author{
В.Г. Кановей
}

\begin{abstract}
Предлагается достаточно удобная и пригодная для развития нестандартного анализа теория множеств в стандартном $\in$-языке.

Библиография: 5 названий.
\end{abstract}

Нестандартные теории множеств составляют одну из двух известных систем оснований нестандартного анализа. (Вторая состоит в использовании нестандартных расширений математических структур в "стандартном" универсуме ZFC.) Типичная нестандартная теория множеств (например, теория внутренних множеств IST Нельсона [1], см. также [2]-[5]) организует универсум множеств таким образом, что объекты обычной математики, назьваемые стандартными, сосуществуют и взаимодействуют с нестандартными объектами (например, бесконечно мальми числами). При этом класс $\mathbb{S}$ всех стандартных множеств выделяется при помощи неопределяемого предиката стандартности st $x$ (читается: $x$ стандартно). Иньми словами, такие нестандартные теории множеств формулируются в st- $\in$-языке, содержащем st и $\in$ в качестве атомарных предикатов.

В настоящей заметке мы предлагаем теорию множеств в $\in$-языке, достаточно сильную для формализации нестандартного анализа. Она названа упрощенной теорией Хрбачека, или SHST. Говоря кратко, SHST - это теория $\in$-структуры универсума HST, в котором верны аксиомы HST (нестандартной теории множеств Хрбачека, использующей st в языке, см. ниже). Теория SHST доказьвает существование насыщенных элементарных расширений. Другим свойством SHST является сушествование (булевозначной) интерпретации в ZFC такой, что класс всех стандартных множеств интерпретации изоморфен универсуму ZFC. В частности, SHST и ZFC равнонепротиворечивы, и любая теорема SHST о стандартных множествах является теоремой ZFC (о всех множествах).

Главной идеей, на которой основано построение аксиоматической системы SHST, является то, что класс $\mathbb{S}$ (сам по себе вряд ли $\in$-определимый в HST) имеет $\in$-определимую изоморфную копию: класс $\mathbb{V}$ всех фундированных множеств (наблюдение Каваи [4]). Это позволяет заменить $\mathbb{S}$ как “стандартный” универсум на $\mathbb{V}$ и использовать очевидную $\in$-определимость $\mathbb{V}$. Класс $\mathbb{I}$ внутренних множеств (элементарное расширение $\mathbb{S}$ ) также допускает $\in$-определение.

Работа выполнена при поддержке Российского фонда фундаментальных исследований, грант № 98-01-00045, фонда DFG, грант № 436-RUS-17/66/97, а также Университета г. Вупперталь. 
1. Теория Хрбачека. Эта теория была введена Хрбачеком [2]. Улучшенная версия представлена в деталях в [3], однако для удобства мы включаем список аксиом HST с краткими комментариями. Напомним, что HST - теория в st- $\epsilon$-языке; st $x$ означает: $x$ стандартно, a $\mathbb{S}=\{x:$ st $x\}$ обозначает класс всех стандартных множеств. Элементы стандартных множеств назьваются внутренними множествами, int $x$ есть формула $\exists^{\text {st }} y(x \in y)$ ( $x$ внутреннее), a $\mathbb{I}=\{x:$ int $x\}$ - класс всех внутренних множеств. Кванторы $\exists^{\text {st }}$ и $\forall^{\text {int }}$ ниже имеют очевидный смысл: “существует стандартное" и "для всякого внутреннего" .

Аксиомы для универсума - все аксиомы ZFC, кроме аксиом регулярности, степени и выбора. Схемы выделения и подстановки формулируются в st- $\epsilon$-языке.

ТРАнзитивность $\mathbb{I} . \forall^{\text {int }} x \forall y \in x$ (int $y$ ).

РЕГУЛЯРНОСТь НАД $\mathbb{I} . \forall X \neq \varnothing \exists x \in X(x \cap X \subseteq \mathbb{I})$.

$\mathbf{Z F C}^{\text {st }}$. Все формулы вида $\Phi^{\text {st }}(\Phi$ релятивизованная к $\mathbb{S})$, где $\Phi$ - аксиома ZFC.

ПЕРЕнос. Все предложения вида $\Phi^{\mathrm{st}} \Longleftrightarrow \Phi^{\mathrm{int}}$, где $\Phi-$ замкнутая $\in$-формула со стандартными параметрами.

СТАндАРТизАция. $\forall X \exists^{\text {st }} Y(X \cap \mathbb{S}=Y \cap \mathbb{S}$ ) (ко всякому $X$ найдется стандартное $Y$, содержащее те же самые стандартные элементы).

Этих аксиом достаточно, чтобы определить класс $\mathbb{V}$ всех фундированных множеств (т.е. элементов транзитивных множеств $X$ таких, что $\in \uparrow X$ фундировано) и $\in$-изоморфизм $x \mapsto{ }^{*} x: \mathbb{V}$ на $\mathbb{S}\left({ }^{*} x\right.$ определяется как единственное стандартное множество $u$, содержащее все множества вида ${ }^{*} y, y \in x$, и больше никаких стандартных элементов). Следовательно, $\mathbb{V}$ - транзитивный класс, интерпретирующий ZFC и замкнутьй относительно взятия подмножеств. Более того, $x \mapsto{ }^{*} x$ - элементарноевложение (в $\in$-языке) $\mathbb{V}$ в $\mathbb{I}$ по переносу (см. [3, раздел 1]).

В HST кардиналь, ординаль, натуральпные числа являются $\mathbb{V}$-понятиями, так что натуральное число понимается как множество $n \in \mathbb{V}$, которое является натуральным числом в $\mathbb{V}$ (кратко, $\mathbb{V}$-натуральным числом). Через $\omega$ обозначается множество всех натуральных чисел.

Множества, равномощные $n=\{0,1, \ldots, n-1\}$, где $n \in \omega$, назьваются конечными. Множества, равномощные некоторому $x \in \mathbb{V}$, назьваются множсествами стандартного размера или, кратко, СР-множсествами.

Сформулируем две последние существенные аксиомы HST.

НАСЫШЕНность $\mathbb{I}$. Если $\mathscr{X} \subseteq \mathbb{I}-\mathrm{CP}$-множество и $\cap \mathscr{X}^{\prime} \neq \varnothing$ для любого конечного $\mathscr{X}^{\prime} \subseteq \mathscr{X}$ (свойство конечных пересечений), то $\cap \mathscr{X}^{\prime} \neq \varnothing$.

СР-выБор. Справедлива аксиома выбора для случая, когда функция выбора должна быть определена на СР-семействе (непустых) множеств.

Насьщенность позволяет получать разнообразные нестандартные множества. Аксиома СР-выбора частично компенсирует отсутствие полной аксиомы выбора, которая, как и аксиомы степени и регулярности, противоречит HST.

Теорема 1 (см. [3]). Теории HST и ZFC равнонепротиворечивы. Более того, HST имеет булевозначную интерпретацию в ZFC, класс $\mathbb{S}$ которой доказуемо

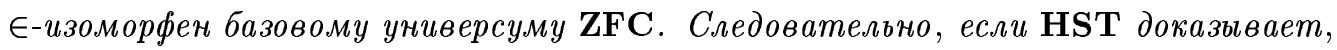


что замкнутая $\in$ формула $\Phi$ истинна в $\mathbb{V}($ или, что равносильно, в $\mathbb{S})$, то $\Phi-$ теорема ZFC.

Неожиданно оказьвается, что класс $\mathbb{I}$ прямо $\in$-определим в HST. Скажем, что множество $x$ квази-внутреннее, когда существует $\omega$-последовательность $\left\{x_{n}\right\}_{n \in \omega}$ такая, что $x \in x_{n+1} \in x_{n}$ для всех $n \in \omega$.

ПРЕДЛОЖЕНИЕ 1 (HST). Классы внутренних и квази-внутренних множеств совпадают.

ДокАЗАтЕльство [3]. Пусть $x \in \mathbb{I}$. Рассуждая в универсуме $\mathbb{I}$, определим индукцией по $k \in{ }^{*} \omega y_{k}=y_{k-1} \cup\left\{y_{k-1}\right\}$, начиная с $y_{0}=x$. Выберем произвольпо $\nu \in *^{*} \omega \backslash \omega$ и положим $x_{n}=y_{\nu-n}$ для всех $n \in \omega$.

Обратная импликация легко следует из регулярности над $\mathbb{I}$.

2. Упрощенная теория Хрбачека. Теория SHST включает следующие группы аксиом (i)-(iv).

(i) Подобно HST все аксиомы ZFC, кроме аксиом регулярности, степени и выбора. (Выделение и подстановка в st- $€$-языке.)

Этого достаточно, чтобы ввести класс $\mathbb{V}$ всех фундированных множеств и доказать его транзитивность.

(ii) Все формулы вида $\Phi^{\mathrm{wf}}$ ( $\Phi$ релятивизованная к $\mathbb{V}=\{x:$ wf $x\}$ ), где $\Phi$ - аксиома ZFC, a wf $x$ говорит: “ $x$ фундировано”.

Далее, пусть ${ }^{q} \mathbb{I}$ обозначаетв $\in$-язьке класс всех квази-внутренних множсеств (см. выше). Мы добавляем

(iii) аксиомы транзитивности класса ${ }^{q} \mathbb{I}$, регулярности над ${ }^{q} \mathbb{I}$, насыщенности ${ }^{q} \mathbb{I}$ и СРвыбора - как в теории HST, но для ${ }^{q} \mathbb{I}$.

Что касается переноса, то непосредственно взять его формулировку из HST нельзя: SHST не обеспечивает никакого подходящего вложения $\mathbb{V}$ в ${ }^{q} \mathbb{I}$. Однако, следующая формулировка достаточно приемлема.

(iv) УПрошЕнныЙ ПЕРенос. Все формулы вида $\Phi^{\text {wf }} \Longleftrightarrow \Phi^{q \text {-int }}$, где $\Phi$ - замкнутая $\epsilon$-формула с параметрами из $\omega$. (Вряд ли можно вовлечь больше параметров дело в том, что $\mathbb{V} \cap \mathbb{I}=H \omega$ (наследственно конечные множества) в HST, но параметры из $H \omega$ сводятся к $\omega$.)

(З десь ${ }^{q \text {-int }}$ означает релятивизацию к ${ }^{q}$.) Эта аксиома нуждается в комментарии, так как сразу неясно, что $\omega \subseteq q \mathbb{I}$. Сценарий состоит в том, что сначала упрощенньй перенос принимается в беспараметрической версии, откуда легко следует, что $q \mathbb{I}-$ транзитивная $\in$-модель $\mathbf{Z F C}$, значит, $\omega \subseteq q \mathbb{I}$. Теперь принимаем упрощенный перенос полностью.

Заметим, что SHST - подтеория $\in$-части HST. (Чтобы доказать упрощенный перенос в HST, мы проверяем в HST, что ${ }^{*} x=x$ для всех $x \in \omega$ индукцией по $x$; тогда $\Phi^{\mathrm{wf}} \Longleftrightarrow \Phi^{\text {int }}$, ибо $x \mapsto{ }^{*} x$ есть элементарное вложение $\mathbb{V}$ в $\mathbb{I}$.) Таким образом, SHST удовлетворяет теореме 1. Следующая лемма показывает, что SHST обеспечивает существование элементарных расширений.

Лемма 1 (SHST). Для всякого транзитивного $X \in \mathbb{V}$ имеется транзитивное ${ }^{*} X \in{ }^{q} \mathbb{I}$ и әлементарное вложсение $\langle X ; \in\rangle$ в $\left\langle{ }^{*} X ; \epsilon\right\rangle$. 
ДокАЗАТЕльство. Перенос и насыщенность SHST дают транзитивное множество ${ }^{*} X \in{ }^{q} \mathbb{I}$ такое, что структуры $\langle X ; \epsilon\rangle$ и $\left\langle{ }^{*} X ; \epsilon\right\rangle$ элементарно эквивалентны. Построим элементарное вложение $\langle X ; \epsilon\rangle$ в $\left\langle{ }^{*} X ; \epsilon\right\rangle$.

По выбору ${ }^{*} X$ и насьшенности $q \mathbb{I}$ если $n \in \omega$, то ко всякому кортежу $\left\langle x_{1}, \ldots, x_{n}\right\rangle \in X^{n}$ имеется кортеж $\left\langle r_{1}, \ldots, r_{n}\right\rangle \in *^{*} X^{n}$ такой, что

(А) для любой $\in$-формулы (в этом доказательстве формулы понимаются как кортежи определенного вида) $A(\cdot, \ldots, \cdot), A\left(x_{1}, \ldots, x_{n}\right)$ истинно в $\langle X ; \in\rangle$ тогда и только тог да, ког да $A\left(r_{1}, \ldots, r_{n}\right)$ истинно в $\left\langle{ }^{*} X ; \epsilon\right\rangle$.

Согласно аксиоме СР-выбора имеется взаимно-однозначное сохраняющее длину кортежей отображение $f: X^{<\omega} \rightarrow\left({ }^{*} X\right)^{<\omega}$ такое, что $(\mathrm{A})$ выполнено для $\left\langle x_{1}^{\prime}, \ldots, x_{n}^{\prime}\right\rangle=$ $f\left(\left\langle x_{1}, \ldots, x_{n}\right\rangle\right)$, каков бы ни был кортеж $\left\langle x_{1}, \ldots, x_{n}\right\rangle \in X<\omega$. Понятно, что $f(\langle x\rangle)=$ $\langle\phi(x)\rangle$, где $\phi: X \rightarrow^{*} X$ есть взаимно-однозначная функция.

Если $D \subseteq X$ конечно, а $F$ - конечное множество $\in$-формул, то пусть $\Pi_{D F} \in q \mathbb{I}$ есть множество всех взаимно-однозначных отображений $\pi \in q \mathbb{I}, \pi:{ }^{*} X$ на ${ }^{*} X$ таких, что для любой $\in$-формулы $A\left(v_{1}, \ldots, v_{n}\right) \in F$ и всех $x_{1}, \ldots, x_{n} \in D$ имеет место

(Б) в * $X$ истинно, что $A\left(\pi\left(\phi\left(x_{1}\right)\right), \ldots, \pi\left(\phi\left(x_{n}\right)\right)\right) \Longleftrightarrow A\left(r_{1}, \ldots, r_{n}\right)$, где $\left\langle r_{1}, \ldots, r_{n}\right\rangle$ $=f\left(\left\langle x_{1}, \ldots, x_{n}\right\rangle\right)$.

Заметим, что множества $\Pi_{D F}$ непусты по выбору ${ }^{*} X$ и $f$. (Например, если $F$ содержит всего одну формулу $A$, просто возьмем биекцию $\pi:{ }^{*} X$ на ${ }^{*} X$ такую, что $\pi\left(\phi\left(x_{i}\right)\right)=r_{i}$ для всех $i$.) Кроме того, семейство всех множеств $\Pi_{D F}$ имеет стандартный размер и свойство конечных пересечений. (В самом деле, $\Pi_{D_{1} F_{1}} \cap \Pi_{D_{2} F_{2}} \supseteq \Pi_{D_{1} \cup D_{2}, F_{1} \cup F_{2}}$.) Значит, существует взаимно-однозначное отображение $\pi \in{ }^{q} \mathbb{I}, \pi{ }^{*} X \rightarrow{ }^{*} X$, принадлежащее каждому из наших множеств $\Pi_{D F}$, так что (Б) вьполнено для всех $x_{1}, \ldots, x_{n} \in X^{<\omega}$ и всех $\in$-формул $A$. Отсюда легко следует, что $p(x)=\pi(\phi(x))$ - элементарное вложение $\langle X ; \epsilon\rangle$ в $\left\langle{ }^{*} X ; \epsilon\right\rangle$.

3. Развитие нестандартного анализа в SHST. Неформальпо, класс $\mathbb{V}$ всех фундированных множеств отождествляется со "стандартным" математическим универсумом. Тогда, поскольку SHST удовлетворяет теореме 1 (как подтеория HST), универсум SHST можно рассматривать как вполне корректно определенное расширение “истинного" универсума $\mathbb{V}$ - подобно тому, как $\mathbb{C}$ есть расширение $\mathbb{R}$. Следовательно, SHST - не просто синтаксический инструмент: мы имеем полную интерпретацию в ZFC.

Как известно, множество $X=V_{\omega+\omega}$, определенное в $\mathbb{V}$, достаточно для построения почти всех математических структур в $\mathbb{V}$. В частности, множества $\mathbb{N}=\omega$ (натуральные числа) и $\mathbb{R}$ принадлежат $\mathbb{V}$.

Лемма 1 приносит транзитивное множество ${ }^{*} X={ }^{*} V_{\omega+\omega} \in{ }^{q} \mathbb{I}$ и элементарное вложение $x \mapsto{ }^{*} x$ структуры $\left\langle V_{\omega+\omega} ; \epsilon\right\rangle$ в $\left\langle{ }^{*} V_{\omega+\omega} ; \in\right\rangle$. (Заметим, что ${ }^{*} V_{\omega+\omega}$ есть ${ }^{q} \mathbb{I}$-аналог $V_{\omega+\omega}$ : фактически, ${ }^{*} V_{\omega+\omega}=V^{*} \omega+{ }^{*} \omega$ в ${ }^{q} \mathbb{I}$.) Легко видеть, что ${ }^{*} n=n \in{ }^{*} \mathbb{N}$ для любого

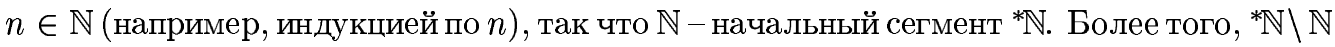
непусто благодаря насыщенности, примененной к семейству множеств $S_{n}=\left\{k \in{ }^{*} \mathbb{N}\right.$ : $k>n\}, n \in \mathbb{N}$. Элементы $* \mathbb{N}$ - это в точности $q \mathbb{I}$-натуральные числа, которые можно назвать, как обычно, гипернатуральными. Числа в ${ }^{*} \backslash \mathbb{N}$ называются бесконечно больиими.

Что касается вещественных чисел (опять в смысле $\mathbb{V}$ ), мы имеем $\mathbb{R} \in V_{\omega+\omega}$ и $\mathbb{R} \subseteq V_{\omega+\omega}$ в $\mathbb{V}$, значит, ${ }^{*} \mathbb{R} \in{ }^{*} V_{\omega+\omega}$ в ${ }^{q} \mathbb{I}$, а ${ }^{*} x \in{ }^{*} \mathbb{R}$ определено для всех $x \in \mathbb{R}$. Элементы ${ }^{*}$, т.е. 
$q \mathbb{I}$-вещественные числа, можно назвать зипервещественными. Теперь можно ввести обычным образом понятия бесконечно больших, бесконечно малых, ограниченных гипервешественных чисел и отношение $\approx$ бесконечной близости.

Лемма 2. Если $x \in{ }^{*} \mathbb{R}$ ограничено, то $x \approx{ }^{*} z$ для некоторого $z \in \mathbb{R}$.

ДоказАтельство. Заметим, что множества $A=\left\{y \in \mathbb{R}:{ }^{*} y \leqslant x\right\}$ и $B=\{y \in \mathbb{R}$ : $\left.{ }^{*} y>x\right\}$ непусты благодаря ограниченности $x$. Эти множества принадлежат $\mathbb{V}$, поскольку этот класс замкнут относительно образования подмножеств. Рассуждая в $\mathbb{V}$, мы находим число $z$, являющееся либо наибольшим в $A$, либо наименьшим в $B$.

Это простое рассуждение демонстрирует возможности SHST. Что касается таких более сложных примеров, как мера Лёба и “гиперконечная" дескриптивная теория множеств, мы сошлемся на [3, 2.2 и 2.3], гдепоказано, как проводить типичные "нестандартные" выкладки в рамках похожих систем.

\section{СПИСОК ЦИТИРОВАННОЙ ЛИТЕРАТУРЫ}

[1] Nelson E. Internal set theory: a new approach to nonstandard analysis // Bull. Amer. Math. Soc. 1977. V. 83. P. 1165-1198.

[2] Hrbaček K. Axiomatic foundations for nonstandard analysis // Fund. Math. 1978. V. 98. P. 1-19.

[3] Kanovei V., Reeken M. Mathematics in a nonstandard world // Math. Japonica. 1997. V. 45. № 2. P. 369-408; № 3. P. 555-571.

[4] Kawaï T. Nonstandard analysis by axiomatic methods // Southeast Asia Conference on Logic (Singapore 1981). Studies in Logic and Foundations of Math. V. 111. Amsterdam: North-Holland, 1983. P. 55-76.

[5] Кусраев А. Г., Кутателадзе С. С. Нестандартные методы анализа. Новосибирск: Наука, 1990.

Московский государственный университет им. М.В. Ломоносова

Поступило

E-mail: kanovei@mech.math.msu.su

16.09.1998 